\title{
Crossmodal facilitation of masked visual target identification
}

\author{
Mary Kim Ngo and Charles Spence \\ University of Oxford, Oxford, England
}

\begin{abstract}
In the present study, participants identified the location of a visual target presented in a rapidly masked, changing sequence of visual distractors. In Experiment 1, we examined performance when a high tone, embedded in a sequence of low tones, was presented in synchrony with the visual target and observed that the high tone improved visual target identification, relative to a condition in which a low tone was synchronized with the visual target, thus replicating Vroomen and de Gelder's (2000, Experiment 1) findings. In subsequent experiments, we presented a single visual, auditory, vibrotactile, or combined audiotactile cue with the visual target and found similar improvements in participants' performance regardless of cue type. These results suggest that crossmodal perceptual organization may account for only a part of the improvement in participants' visual target identification performance reported in Vroomen and de Gelder's original study. Moreover, in contrast with many previous crossmodal cuing studies, our results also suggest that visual cues can enhance visual target identification performance. Alternative accounts for these results are discussed in terms of enhanced saliency, the presence of a temporal marker, and attentional capture by oddball stimuli as potential explanations for the observed performance benefits.
\end{abstract}

Inspired by the pioneering crossmodal research of O'Leary and Rhodes (1984), Vroomen and de Gelder (2000) reported a study in which they explored whether the perceptual organization of an auditory stimulus stream would modulate the perceptual organization of simultaneously presented visual information. Vroomen and de Gelder were particularly interested in the question of whether the organization of a sequence of tones could facilitate the identification of a visual target in a rapidly presented sequence of visual displays. The visual target in their experiment was presented in one of the four corners of a visual display. The participants had to indicate in which corner the target appeared. In one condition, the sequence of visual displays was accompanied by a sequence of four low tones (LLLL), whereas, in another condition, the visual displays were accompanied by a sequence of three low tones and a high tone (LLHL). Importantly, the third out of every four visual displays in the sequence always contained a target (see Figure 1) and was, thus, synchronized either with a low tone in the LLLL tone sequence or with a high tone in the LLHL tone sequence (see Vroomen \& de Gelder, 2000, Experiment 1).

Vroomen and de Gelder (2000) reported that their participants responded significantly more rapidly and accurately when identifying the visual target if the displays were accompanied by the LLHL sequence, as compared with the LLLL sequence. They attributed this crossmodal facilitation of participants' masked visual target identification performance following the presentation of a unique sound to the segregation taking place in the auditory stream, which occurred for the LLHL tone sequence, but not for the LLLL tone sequence. Vroomen and de Gelder argued that the participants could clearly differentiate between the low- and high-frequency tones in the LLHL sequence and that this auditory stream segregation carried over to influence their perception of stimuli in the visual modality (cf. O'Leary \& Rhodes, 1984; see also Spence \& Chen, in press; Spence, Sanabria, \& Soto-Faraco, 2007). Vroomen and de Gelder stated that "perceptual evaluation in one modality may then have consequences in other modalities so that coherence is maintained. A sound that segregates in the auditory modality may for that reason provoke segregation in the visual modality" (p. 1590). In the context of crossmodal perceptual organization, Vroomen and de Gelder argued that the segregation of the auditory cue and the visual target display from the distractor displays allowed the participants to access the information contained within the visual target display for longer than in the LLLL sequence trials, thus resulting in enhanced visual target identification performance.

Vroomen and de Gelder (2000, Experiment 5) directly tested the crossmodal perceptual organization account of their results by manipulating the likelihood that the high tone would segregate from the sequence of low tones. In particular, they introduced an LMHL (low-middle-highlow) tone sequence condition with the rationale that the high tone in this sequence would be less abrupt (and noticeable) when it was preceded by the presentation of the

M.K.Ngo, thuan.ngo@psy.ox.ac.uk 


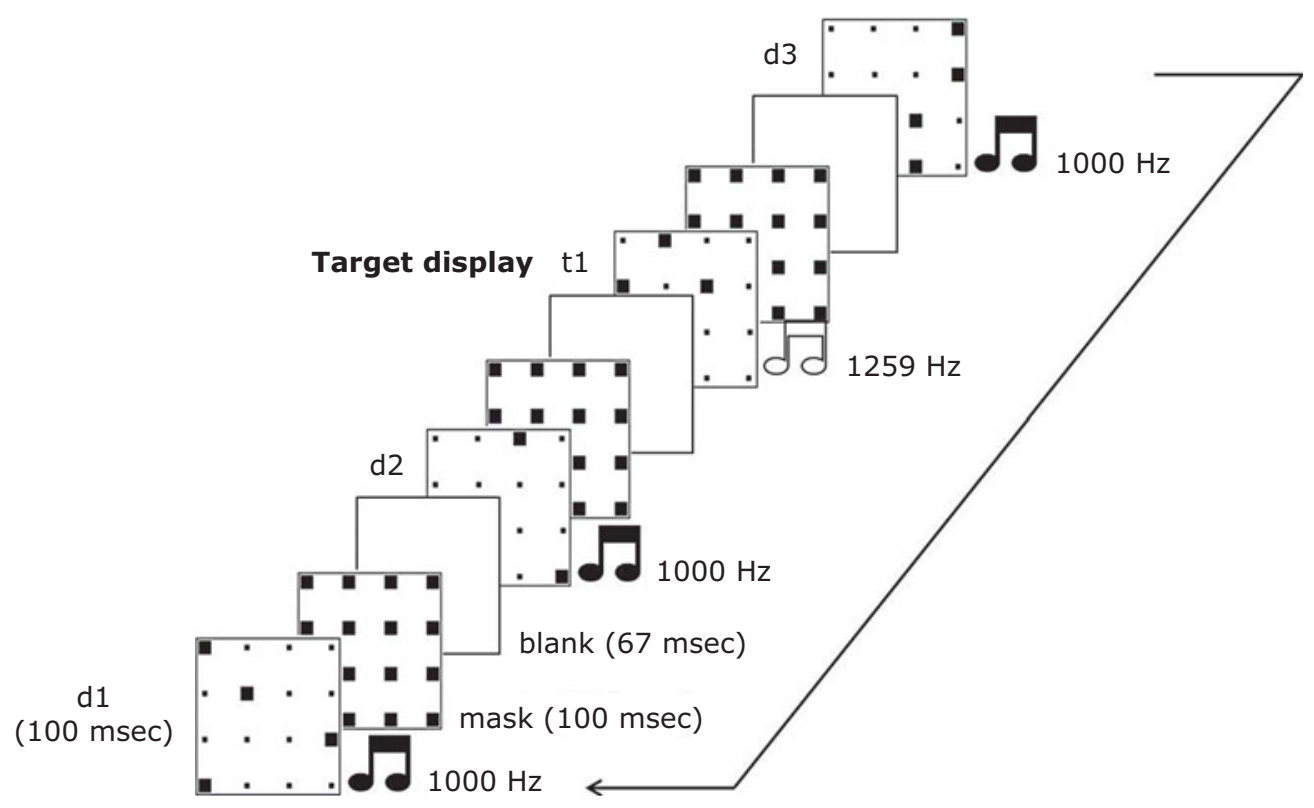

Figure 1. Schematic representation of a sequence of stimulus displays for the LLHL condition in Experiment 1 . The figure shows a sequence of four masked visual displays (d1, d2, t1, and d3), with each display accompanied by a tone. The three distractor displays (d1-d3) are each accompanied by a low (1000-Hz) tone, whereas the target display (t1) is accompanied by a high $(1259-\mathrm{Hz})$ tone.

middle tone (two semitones higher than the low tone) than would the same high tone embedded in the LLHL sequence. Given that abrupt sounds are more likely to segregate than less abrupt sounds (Bregman, 1990), they predicted that the high tone would be less likely to segregate from the LMHL sequence as compared with the LLHL sequence, which in turn would lead to worse performance when the target display was synchronized with the high tone from the LMHL sequence than from when it was synchronized with the high tone from the LLHL sequence. This is indeed what was observed, thus supporting Vroomen and de Gelder's crossmodal perceptual organization account of their data.

The importance of a sound's abruptness in the context of auditory stream segregation can be taken as evidence in support of Vroomen and de Gelder's (2000) crossmodal perceptual organization account. It can also be taken, however, to suggest that any single abrupt cue (i.e., visual, auditory, or tactile) may be, in and of itself, sufficient to enhance participants' visual target identification performance. This may not necessarily be because it allows for better auditory stream segregation (and hence, crossmodal perceptual organization) but, perhaps, because the abruptness of the auditory cue somehow increases the saliency of the synchronous visual target (cf. Anderson \& Mamassian, 2008; Noesselt, Bergmann, Hake, Heinze, \& Fendrich, 2008; Van der Burg, Cass, Olivers, Theeuwes, \& Alais, 2010). Vroomen and de Gelder did not test this possibility, however. Instead, in all six of the experiments that they reported, they used an auditory sequence with the high tone always embedded in a sequence of lower tones. Thus, whether the effect of the tone's abruptness worked to modulate the crossmodal perceptual organization of the auditory and visual information or whether, instead, it affected some other mechanism responsible for the observed performance benefits could not be dissociated. Moreover, the "abruptness" of the sound, in the sense that the term was used by Vroomen and de Gelder, is ambiguous. In the context of the stream of auditory tones used in their study, the abruptness of the high tone referred to the magnitude of the frequency change between the synchronized high tone and the distracting low tones. The abruptness of a cue, in its traditional sense, though, is used to refer to the rise/decay time of the cue. Because there appears to be a discrepancy between the traditional use of the term abrupt to describe the sudden onsets of cues and that used by Vroomen and de Gelder to describe the change in frequency between high and low tones, it is important to note that, in our study, we refer to any single onset cue as abrupt and the high tone within a sequence of other low tones as a frequency change.

In another experiment, Vroomen and de Gelder (2000; Experiment 4) demonstrated that even when the temporal structure or order of the LLHL tone sequence was disrupted by varying the number of low tones (i.e., distractors) preceding the presentation of the high tone (i.e., the target) so that the occurrence of the high tone was made unpredictable, they still found a significant facilitation of participants' visual target identification performance when the high tone was presented at the same time as the visual target. Vroomen and de Gelder concluded that the synchrony of the auditory cue with the visual target was a key factor in eliciting the crossmodal performance benefits that were observed. Similarly, recent research by Van der Burg, Olivers, Bronkhorst, and Theeuwes (2008b, 2009; see also Ngo \& Spence, 2010) has demonstrated that the presentation of temporally synchronous auditory and 
tactile cues can significantly improve the identification of visual targets in a visual search paradigm. The participants in the latter studies had to search for a horizontal or vertical line segment presented among a varying number of tilted distractor line segments. The color of the target and distractors changed regularly from red to green (or vice versa) on a given trial. Either the onset of the auditory or vibrotactile cue was synchronized with the color change of the visual target, or else no cue was presented. The presentation of the temporally synchronous cues gave rise to a significant reduction in search latencies (in excess of $1,000 \mathrm{msec}$ ) for visual targets.

On the basis of the results of Vroomen and de Gelder's (2000) experiments and those reported in Van der Burg et al.'s (2010; Van der Burg et al., 2008b, 2009; see also Ngo \& Spence, 2010) studies, it appears as though the necessary conditions for achieving a significant improvement in participants' visual target identification involve the presentation of cues that are abrupt and temporally synchronous with the onset of the visual target. If the abruptness and temporal synchrony of the cue are at stake, it seems logical to predict that a single, temporally synchronous cue (be it auditory, tactile, or even visual, for that matter) should suffice to produce the same, if not larger, improvements in performance as a high tone embedded in a sequence of low tones. Vroomen and de Gelder did not test this possibilitypresumably, because their study was framed in the context of crossmodal perceptual organization.

In fact, to date, no studies have directly attempted to replicate Vroomen and de Gelder's (2000) important findings with regard to crossmodal perceptual organization or tested the possibility that a single cue, when presented in synchrony with the target display, could lead to performance improvements similar to those observed in the presence of the stream-segregated high tone embedded in the sequence of low tones. If a single, temporally synchronous cue (auditory, tactile, or visual) were found to facilitate participants' visual target identification performance, this would suggest that one does not necessarily need to involve the notion of auditory stream segregation, or crossmodal perceptual organization, in order to explain the reported performance benefits. Perhaps, by itself, a single cue might suffice to produce the freezing effect and, hence, enhance the saliency of the neural representation of the visual target. However, if a single cue were not to give rise to any facilitation of participants' visual target identification performance, this would then provide support for the idea that it is the perceptual organization of the auditory and visual streams that critically underlies the crossmodal facilitatory effects that were observed in Vroomen and de Gelder's study. The first goal of the present study was therefore to test these two possibilities, using an experimental setup identical to that in Vroomen and de Gelder (Experiment 1).

\section{EXPERIMENT 1}

\section{Method}

Participants. Eighteen participants ( 15 of them female; age range $=18-36$ years; mean age $=26$ years $)$ took part in this experi- ment. All of the participants reported normal or corrected-to-normal vision and normal hearing. The experiment took approximately $20 \mathrm{~min}$ to complete. The participants received a $£ 5$ (U.K. Sterling) gift voucher in return for taking part in the experiment.

Apparatus and Stimuli. All of the experiments were conducted in a dimly lit, anechoic chamber. The participants sat in a chair approximately $55 \mathrm{~cm}$ from a 17 -in. CTX PR711F visually flat CRT computer monitor (CTX, Watford, U.K.; screen refresh rate $=$ $75 \mathrm{~Hz}$ ). The visual display consisted of a $4 \times 4$ array of quasirandomly flickering white dots (each dot was $4 \times 4$ pixels in size), and the entire array measured $4.2 \times 4.2 \mathrm{~cm}$ in total. The flickering display was created by presenting a series of visual stimuli in rapid succession. Each visual display contained 4 uniquely positioned white dots, which made up the full matrix of 16 dots when the various 4-dot displays were overlaid.

The third stimulus in each quartet of displays always contained four dots positioned so as to form a diamond. This diamond constituted the target stimulus that the participants had to detect and whose location they had to indicate. The target display always appeared in one of the four corners of the matrix: top left, top right, bottom left, or bottom right. The participants made their responses using the numerical keypad of a standard QWERTY keyboard by pressing "9" if the target appeared in the top right, "7" if the target appeared in the top left, " 3 " if the target appeared in the bottom right, or " 1 " if the target appeared in the bottom left corner of the display. Note that the third (target) display in each four-display sequence was the only one to contain dots that were arranged so as to form a diamond (i.e., the dots never formed a diamond in any of the distractor displays).

Each 4-dot display (including the target display) was presented for $100 \mathrm{msec}$ (i.e., for six screen refreshes) and was immediately followed by a mask containing all 16 dots (i.e., the entire matrix), which was also presented for $100 \mathrm{msec}$. Immediately after the presentation of the mask, a blank screen was presented for $67 \mathrm{msec}$. This cycle was repeated continuously with no interruption until either the participant made a response or 10 full cycles had been presented. If the participant had not made a response by the end of the 10th cycle, the next trial began automatically. Each trial began when the participant pressed the space bar and ended either when the participant made a response or when the 10th display cycle had been presented.

The auditory stimuli in Experiment 1 consisted of sequences of either four $1000-\mathrm{Hz}$ low tones (denoted as LLLL) or three low tones and a single, $1259-\mathrm{Hz}$ high tone (denoted as LLHL, with the high tone always presented in the third position of each four tones and in temporal synchrony with the target display; $44.1-\mathrm{kHz}$ sampling rate, 16 bits). Note that these tones were presented at exactly the same frequencies as those used in Vroomen and de Gelder's (2000) original study. The tones were $100 \mathrm{msec}$ long (including a 5 -msec fade-in and fade-out to avoid clicks) and were presented from two Dell A215 PC loudspeaker cones (Dell Inc., U.K.), one placed $16^{\circ}$ to either side of the center of the visual display. The tones appeared to have been presented from the center of the visual display.

Design and Procedure. The tone sequence (LLLL or LLHL) was varied randomly on a trial-by-trial basis, with each tone sequence occurring equiprobably within the experimental session. The target was presented 20 times in each of the four corners of the display with both the LLLL and the LLHL sequences, giving rise to a total of 160 pseudorandomly ordered trials in each participant's experimental session.

Because auditory stream segregation takes time to build up (see, e.g., Bregman, 1990), the participants listened to a series of LLLL and LLHL tone sequences before starting the main experimental session (just as in Vroomen \& de Gelder's, 2000, original study). Each sequence alternated between four and eight repetitions (with no gaps between the successive sequences) giving rise to a total of four alternations. Thus, the participants heard the LLLL sequence four times in a row, followed by the LLHL sequence four times in a row, and then the LLLL sequence eight times in a row, followed by the LLHL sequence eight times in a row. The entire sequence was repeated four times, with no gaps between any of the tone sequences. 
Table 1

Mean Reaction Times (RTs), Their Standard Errors (SEMs), and Percentages of Errors for the Various Cue Conditions in Experiments 1-5

\begin{tabular}{|c|c|c|c|c|}
\hline \multirow[b]{2}{*}{ Experiment } & \multirow{2}{*}{$\begin{array}{c}\text { Cue } \\
\text { Condition }\end{array}$} & \multicolumn{2}{|c|}{ RT (msec) } & \multirow[b]{2}{*}{ Error $(\%)$} \\
\hline & & $M$ & $\overline{S E M}$ & \\
\hline \multirow[t]{2}{*}{1} & LLLL & 3,613 & 322 & 25 \\
\hline & LLHL & 3,181 & 239 & 19 \\
\hline \multirow[t]{2}{*}{2} & Tone absent & 3,036 & 276 & 30 \\
\hline & Tone present & 2,114 & 268 & 19 \\
\hline \multirow[t]{4}{*}{3} & No cue & 4,273 & 304 & 30 \\
\hline & Auditory & 3,664 & 322 & 25 \\
\hline & Vibrotactile & 3,724 & 286 & 27 \\
\hline & Audiotactile & 3,673 & 290 & 29 \\
\hline \multirow[t]{2}{*}{4} & Box absent & 2,767 & 265 & 28 \\
\hline & Box present & 2,684 & 325 & 19 \\
\hline \multirow[t]{3}{*}{5} & No cue & 3,017 & 385 & 28 \\
\hline & Auditory & 2,265 & 434 & 13 \\
\hline & Visual & 2,495 & 348 & 16 \\
\hline
\end{tabular}

Note-L, low tone; $\mathrm{H}$, high tone.

During this preexperimental session, the participants listened to the tones, which were presented in synchrony with masks (i.e., displays containing all 16 dots) and were told not to make any response. Each mask was presented for $200 \mathrm{msec}$, followed by a blank display for $67 \mathrm{msec}$. The participants were then given 16 practice trials before beginning the main experimental session, with half of the trials presented with the LLLL sequence and the remainder presented with the LLHL sequence. The participants were instructed to identify the location of the target diamond as rapidly and accurately as possible. They were also told that the target would always be presented in the third visual display out of each four-display quartet.

\section{Results}

The reaction time (RT) and accuracy of the participants' responses were measured. RTs were measured from the onset of the first target display until the participant responded. RTs and percentages of correct responses were subjected to an ANOVA with tone sequence (LLLL vs. LLHL) as the within-participants factor (the RT and error data for each condition are also reported for completeness in Table 1). The ANOVA revealed a significant main effect on the percentage of correct responses $[F(1,17)=6.50$, $\left.p=.02, \eta^{2}=.28\right]$. That is, the participants responded more accurately when the visual displays were accompanied by the LLHL tone sequence ( $M=81 \%$ correct) than when they were presented with the LLLL tone sequence ( $M=75 \%$ correct; see Figure 2$)$. The participants also responded significantly more rapidly when the displays were presented simultaneously with the LLHL tone sequence $(M=3,181 \mathrm{msec})$ than when they were presented with the LLLL tone sequence $(M=3,613 \mathrm{msec})[F(1,17)=9.57$, $\left.p=.007, \eta^{2}=.36\right]$.

\section{Discussion}

The results of Experiment 1 demonstrate that the participants identified the visual targets both significantly more quickly and more accurately when they were accompanied by a temporally synchronous high tone in the LLHL tone sequence, as compared with when they were accompanied by a low tone in the LLLL tone sequence. Thus, we replicated Vroomen and de Gelder's (2000, Experiment 1) basic findings in terms of significant effects being reported in both the speed and accuracy of participants' target identification responses when an experimental setup was used that was more or less identical to theirs. ${ }^{1}$ Having replicated their basic result (note that this is the first replication of Vroomen and de Gelder's findings in the decade since their study was originally published), we next went on to examine whether or not a single tone presented in synchrony with the visual target would lead to a performance benefit similar to that observed with the LLHL tone sequence.

\section{EXPERIMENT 2}

\section{Method}

Eighteen participants from the University of Oxford (12 of them female), ranging in age from 18 to 39 years (mean age $=26$ years), took part in this experiment. The experimental setup for Experiment 2 was essentially the same as that in Experiment 1, with the following exceptions. First, instead of having tones presented on each trial, only half of the trials were tone-present trials, whereas the remainder were tone-absent trials. Second, instead of having four synchronous tones presented with each of the 4 four-dot displays (as in Experiment 1), only a single 1259-Hz tone was presented simultaneously with the target display on the tone-present trials. The presence versus absence of the tone was varied on a trial-by-trial basis.

\section{Results}

RTs and percentages of correct responses were once again subjected to separate ANOVAs with tone presence (absent vs. present) as the within-participants factor (the RT and error data for each condition are also reported for completeness in Table 1). The ANOVA on the mean RT data revealed a significant main effect $[F(1,17)=$ $\left.32.87, p<.001, \eta^{2}=.66\right]$. The participants responded significantly more rapidly on the tone-present trials ( $M=2,114 \mathrm{msec})$ than on the tone-absent trials $(M=$ 3,036 msec; see Figure 3). There was also a significant main effect of tone presence on the percentages of correct responses $\left[F(1,17)=12.29, p=.003, \eta^{2}=.42\right]$, with

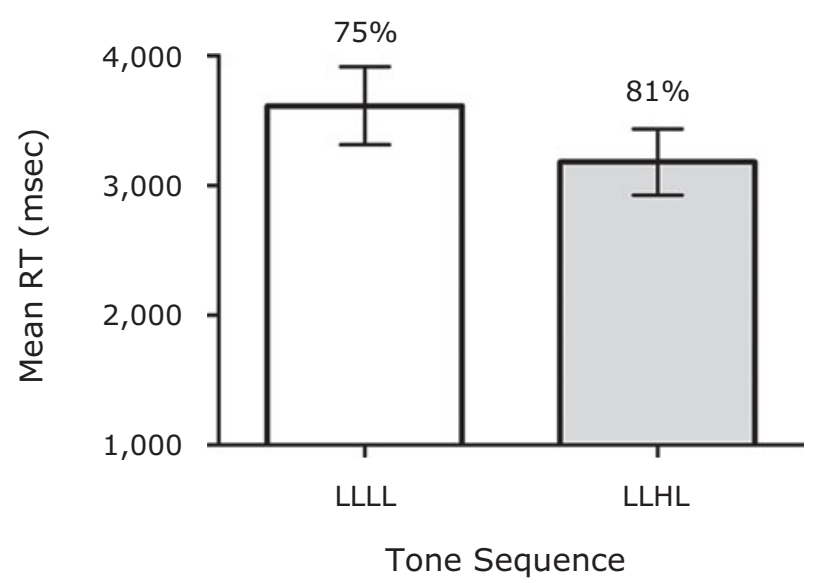

Figure 2. Mean reaction times (RTs, in milliseconds) for LLLL and LLHL conditions in Experiment 1. The error bars represent the standard errors of the means. The mean percentage of correct responses for each tone sequence is shown above the corresponding error bar. 


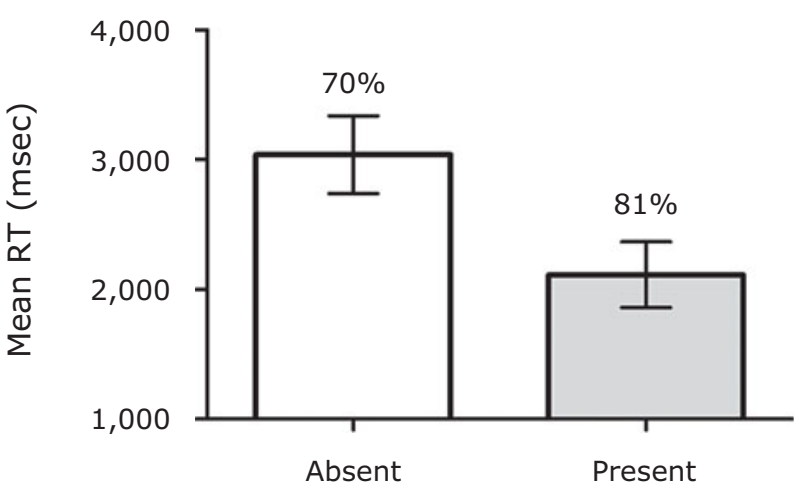

Tone

Figure 3. Mean reaction times (RTs, in milliseconds) for toneabsent and tone-present conditions in Experiment 2. The error bars represent the standard errors of the means. The mean percentage of correct responses for each condition is shown above the corresponding error bar.

the participants responding more accurately on the tonepresent trials $(M=81 \%)$ than on the tone-absent trials $(M=70 \%$; see Figure 3).

\section{Discussion}

The results of Experiment 2 revealed that a single tone, when presented in synchrony with a visual target, led to significantly faster and more accurate visual target identification performance. Interestingly, the effect sizes when a single tone was presented in Experiment 2 were larger for both the RT and accuracy data than were those for the LLHL tone sequence in Experiment 1. Moreover, the average reduction in RTs was larger $(M=922 \mathrm{msec})$ when the single tone was presented than when the tone was embedded in the LLHL sequence $(M=432 \mathrm{msec})$. These results therefore support the prediction that a single, temporally synchronous cue would lead to an improvement in participants' visual target identification performance that was at least equivalent to (and in this case, much larger than) that reported in Vroomen and de Gelder's (2000) previous study. It is possible, then, that rather than crossmodal perceptual organization, another mechanism may underlie the crossmodal facilitation effect observed in the present study and that in Vroomen and de Gelder.

The results of Experiment 2 provide initial evidence to support the notion that the abruptness and temporal synchrony of the cue were vital in enhancing participants' visual target identification performance. Given that several recent studies have shown that tactile cues produce improvements in participants' visual target identification performance comparable to those observed with auditory cues (see Ngo \& Spence, 2010; Van der Burg et al., 2008b, 2009), we next went on to investigate whether similar visual target performance benefits might be seen using vibrotactile, rather than auditory, cues in the masked visual target identification paradigm introduced by Vroomen and de Gelder (2000). At the same time, we also wanted to investigate whether multisensory (audiotactile) cues would give rise to performance benefits that were any larger than those seen following unimodal (auditory or tactile) cuing (cf. Santangelo, Ho, \& Spence, 2008; Spence \& Santangelo, 2009).

\section{EXPERIMENT 3}

\section{Method}

Sixteen participants from the University of Oxford ( 9 of them female), ranging in age from 18 to 36 years (mean age $=25$ years), took part in this experiment. The experimental setup was similar to that used in Experiment 2, in that it included a tone-absent and a tone-present condition. In addition, however, unimodal tactile and bimodal audiotactile cue conditions were also included in the design. The tactile cue consisted of a $100-\mathrm{msec}$ vibration presented by means of a single VBW32 (Audiological Engineering Corporation, Somerville, MA) tactor attached to the back of the participants' left hand. The participants placed their left hand in the middle in front of the space bar of the keyboard, which was centered in front of the computer screen. The intensity of the vibrotactile stimulus was subjectively matched to that of the auditory tone prior to conducting the actual experiment by four volunteers, who did not take part in the main experiment. The four cue conditions (no cue, auditory, tactile, or audiotactile) were interleaved randomly within each block of trials. Each of the four blocks of trials consisted of 80 trials, with each of the four cue conditions and target positions occurring equiprobably and in random order within each block of trials.

\section{Results}

RTs and percentages of correct responses were once again subjected to separate ANOVAs with cue type (no cue, auditory, tactile, or audiotactile) as the withinparticipants factor (the RT and error data for each condition are also reported for completeness in Table 1). The ANOVA revealed a significant main effect on the mean RT data $\left[F(3,45)=6.88, p=.003, \eta^{2}=.31\right]$. All three cue types (auditory, tactile, and audiotactile) led to faster responses $(M=3,664,3,724$, and $3,673 \mathrm{msec}$, respectively) than the no-cue condition $(M=4,273 \mathrm{msec}$ ) ( $p=$ $.012, .003$, and .008 , respectively; see Figure 4$)$. The differences in accuracy between the various cue conditions failed to reach significance $[F(3,45)=1.9, p=.14]$.

\section{Discussion}

The results of Experiment 3 demonstrated that although the presentation of a nonvisual cue synchronized with the visual target once again led to faster responses by our participants (thus replicating the results of Experiment 2), there were no differences among the three cue conditions. Thus, auditory, tactile, and audiotactile cues all led to similar performance benefits in terms of improved visual target identification performance, relative to that seen in the no-cue baseline. This finding suggests that the tactile modality can provide an alternative cue modality that may be just as effective as the more typically used auditory cue modality in terms of facilitating participants' performance on visual tasks. What is more, bimodal audiotactile cues did not lead to any further improvements in the participants' performance over that seen in response to the unimodal cues. In fact, of the three cued conditions, the average accuracy for the audiotactile cue condition was numerically somewhat lower $(71 \%)$ than that in either the auditory $(75 \%)$ or the tactile $(73 \%)$ cue condition. Mean 


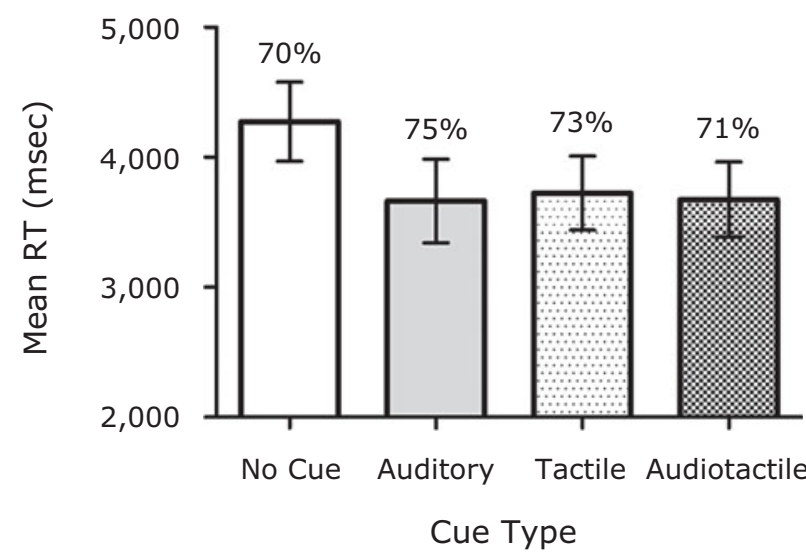

Figure 4. Mean reaction times (RTs, in milliseconds) for the no-cue and the auditory, tactile, and audiotactile cue conditions in Experiment 3. The error bars represent the standard errors of the means. The mean percentage of correct responses for each condition is shown above the corresponding error bar.

RTs were also numerically slightly shorter for the auditory $(3,664 \mathrm{msec})$ than for the audiotactile $(3,673 \mathrm{msec})$ cue condition. Thus, there was no evidence of any speedaccuracy trade-offs in the data. This pattern of results is consistent with that reported recently by Ngo and Spence (2010). They also found that temporally synchronous unimodal auditory and tactile cues were just as effective as bimodal audiotactile cues in (significantly) reducing search latencies for visual targets presented amid a dynamic and cluttered visual display (cf. Spence \& Santangelo, 2009; Van der Burg et al., 2008b, 2009).

\section{EXPERIMENT 4}

The results of Experiments 1-3 showed that unimodal and multisensory nonvisual cues, when presented simultaneously with the visual target, resulted in improved visual target identification in terms of both the speed and accuracy of participants' responding. Several recent studies have shown that crossmodal cuing is better than unimodal cuing (see Spence \& Santangelo, 2009, for a recent review). Van der Burg et al. (2008b; Experiment 2B) even found that the presentation of a visual cue did not give rise to any significant improvements in participants' visual target identification performance (see also Noesselt et al., 2008). In Experiment 4, we examined whether a visual cue would give rise to a significant visual target performance benefit, using Vroomen and de Gelder's (2000) rapidly masked serial visual presentation paradigm. If the visual cue did not lead to any improvements in participants' visual target identification performance, this would suggest that crossmodal cues are necessary for facilitating performance on this visual target identification task. If, on the other hand, the presentation of a visual cue was to significantly facilitate participants' visual target identification performance, this would instead suggest that the benefit of presenting a temporally synchronous cue need not be crossmodal. This might suggest, instead, that the cue simply provides a temporal marker for the visual tar- get and, hence, its modality is irrelevant (Watson, Humphreys, \& Olivers, 2003).

\begin{abstract}
Method
Sixteen participants from the University of Oxford (11 of them female), ranging in age from 20 to 34 years (mean age $=24$ years), took part in this experiment. The experimental setup was nearly identical to that in Experiment 2, with the sole exception that rather than a tone, on half of the trials, the target was presented together with a light gray outline box surrounding the $4.2 \times 4.2 \mathrm{~cm}$ grid in which the target appeared (i.e., the visual cue outlined the entire visual display; see Figure 5). The presence versus absence of this visual cue was varied on a trial-by-trial basis, just as for the tone presented in Experiment 2.
\end{abstract}

\section{Results}

RTs and percentages of correct responses were subjected to separate ANOVAs with box presence (absent vs. present) as the within-participants factor (the RT and error data for each condition are also reported for completeness in Table 1). The ANOVA revealed a significant main effect of the presence of the visual cue on the accuracy of the participants' responses $\left[F(1,15)=6.83, p=.02, \eta^{2}=\right.$ .31], with more accurate responding being observed when it was present $(M=81 \%)$ than when it was absent $(M=$ $72 \%$ ). There was, however, no significant effect on mean RTs $(F<1$, n.s.; see Figure 6).

\section{Discussion}

Although the participants were not significantly faster at identifying the visual target in the presence of the visual cue box, as compared with when it was absent, they were significantly more accurate in their target identification responses. Thus, visual cues, when presented in synchrony with the visual target, led to an improvement in the participants' visual target identification performance, at least in terms of the accuracy of their responding. A closer look at the effect sizes from this experiment and those reported in Experiment 2 also showed that the effect size for the presence of the single tone in Experiment 2 was larger than the

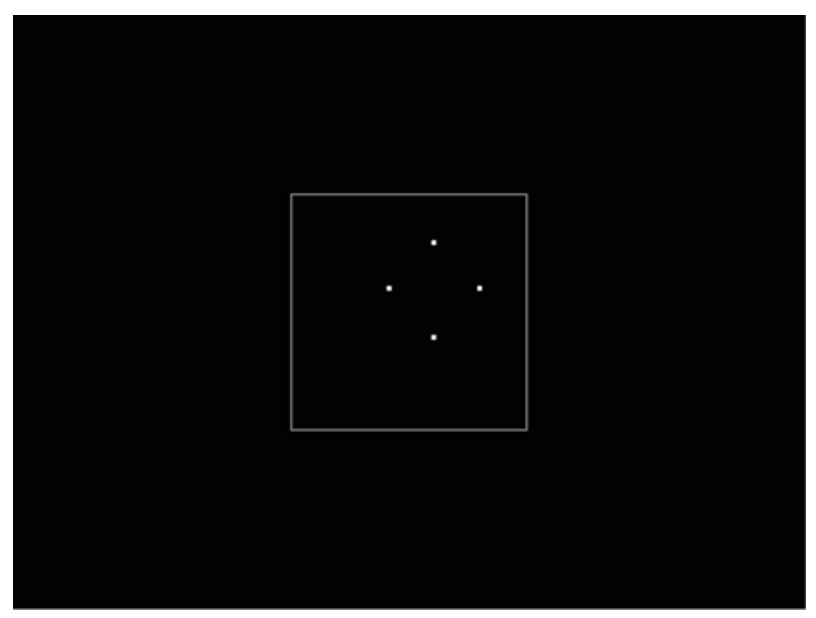

Figure 5. Illustration of the light gray outline of a box surrounding the visual target display presented on the computer screen in Experiment 4. 


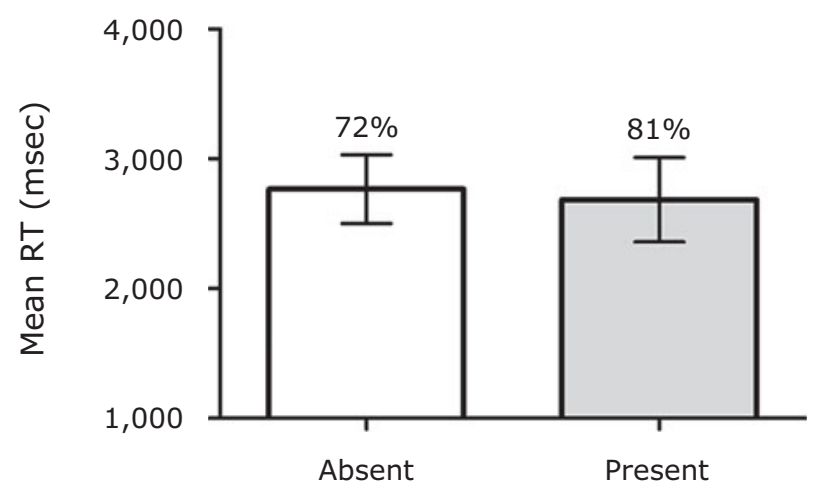

Visual Cue

Figure 6. Mean reaction times (RTs, in milliseconds) for the box-absent and box-present conditions in Experiment 4. The error bars represent the standard errors of the means. The mean percentage of correct responses for each condition is shown above the corresponding error bar.

effect size observed here in Experiment 4. That is, the auditory cue in Experiment 2 appeared to be more effective at facilitating the participants' visual target identification performance than was the visual cue in Experiment 4. In fact, the improvement in the participants' performance in the presence of the auditory cue in Experiment 2 was a 922-msec reduction in average RTs and an $11 \%$ increase in the accuracy of the participants' responses, whereas the presence of the visual cue in Experiment 4 led to only an $83-\mathrm{msec}$ reduction in average RTs and a 9\% increase in accuracy.

The provisional conclusion is that although temporally synchronous visual cues do lead to some improvement in participants' visual target identification performance in the Vroomen and de Gelder (2000) task, the optimal cue may need to be nonvisual (i.e., crossmodal) in order to maximize any potential facilitatory effects observed for visual target perception and identification (cf. Watanabe \& Shimojo, 2001). Although there may be an additional benefit for participants' visual target identification performance resulting from the presentation of the auditory (i.e., crossmodal), as compared with the visual (i.e., intramodal), cue, it is important to note the betweenparticipants nature of the comparison leading to this conclusion. It is also important to note that the mean RTs from the no-cue conditions in Experiments 2 and 4 (i.e., a condition that was exactly the same in the two experiments) differed by nearly $300 \mathrm{msec}$, pointing to the fact that there may have been individual differences between the participants tested in the two experiments. Therefore, in order to be certain about the relative improvements in the participants' visual target identification performance following the presentation of either the auditory or the visual cue, we used a within-participants design and included both cue types in Experiment 5. In this way, we were able to directly compare the participants' performance on the visual target identification task when they were exposed to both the auditory and visual cue conditions.

\section{EXPERIMENT 5}

\section{Method}

Twelve participants from the University of Oxford (5 of them female; age range $=19-32$ years; mean age $=25$ years) took part in this experiment. The experimental setup was similar to that in Experiment 4, with the exception that the participants completed one block of 80 trials in which no cue was presented with the visual target and a second block of 160 trials in which the visual target was presented with the synchronized $1259-\mathrm{Hz}$ tone on half of the trials and the synchronous visual cue (the light gray outline of a box) on the remaining half of the trials. The auditory and visual cues were interleaved randomly within the block of trials, and the order of presentation of the blocks was counterbalanced across participants.

\section{Results}

RTs and percentages of correct responses were subjected to separate ANOVAs with cue type (no cue, auditory, or visual) as the within-participants factor (the RT and error data for each condition are also reported for completeness in Table 1). The ANOVA revealed a significant main effect of cue type on the accuracy of the participants' responses $\left[F(2,22)=18.49, p<.001, \eta^{2}=.63\right]$. The participants responded significantly more accurately when the visual target was presented with either the auditory $(M=87 \%)$ or the visual $(M=84 \%)$ cue than when no cue $(M=72 \%)$ was presented ( $p<.001$ and .001 , respectively; see Figure 7). In this experiment, there was no significant difference in accuracy between the auditory and visual cue conditions $(p=.28)$. There was a significant main effect of cue type on RTs $\left[F(2,22)=7.40, p=.003, \eta^{2}=\right.$ $.40]$, with participants responding more rapidly following the presentation of either the tone $(M=2,265 \mathrm{msec})$ or the box $(M=2,495 \mathrm{msec})$ cue than in the no-cue $(M=$ $3,017 \mathrm{msec})$ condition ( $p=.003$ and .034 , respectively). There was no significant difference in RTs between the auditory and visual cue conditions $(p=.24)$.

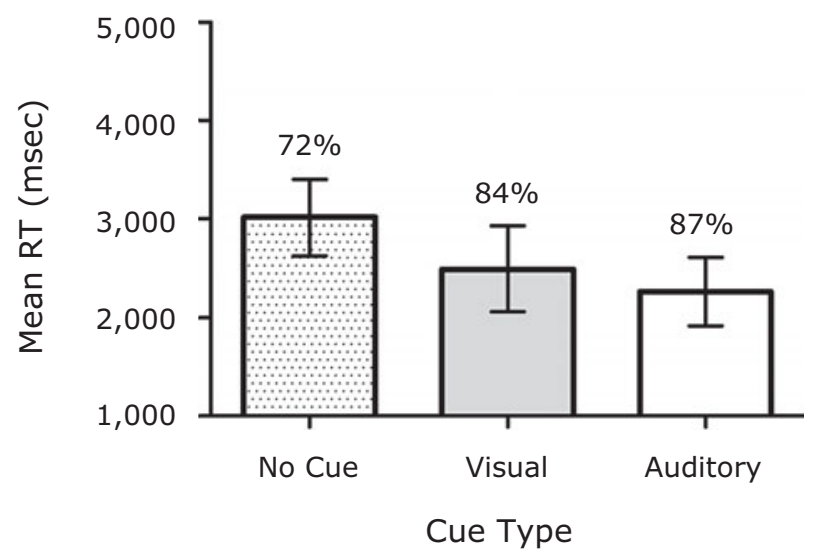

Figure 7. Mean RT (in milliseconds) for the no-cue, auditory cue, and visual cue conditions of Experiment 5. The error bars represent the standard errors of the means. The mean percentage of correct responses for each condition is shown above the corresponding error bar. 


\section{Discussion}

The results of Experiment 5 revealed that the visual cue, like the auditory cue, was also effective in facilitating the participants' performance on the masked visual target identification task (when compared with performance in a condition in which no cue was presented). This result supports the view that a cue need not be crossmodal in order to elicit a significant improvement in the identification of a visual target. Alternatively, however, the fact that the presence of the synchronous auditory and visual cues were interleaved within one block of trials, whereas the cue-absent trials were presented in a separate block, may have led to the participants' adopting a search strategy, or attentional set, which might have allowed them to capitalize on the temporally informative nature of the synchronous cue, regardless of its sensory modality (see Leber, Kawahara, \& Gabari, 2009). The participants in Experiment 4, on the other hand, may not have fully adopted an attentional set that was responsive to the temporal information provided by the visual cue, since both cue-present and cue-absent trials were interleaved within each block of trials, thus possibly resulting in a partial performance benefit manifesting in an improvement in the accuracy (but crucially, not the speed) of their responding.

\section{GENERAL DISCUSSION}

The results of Experiment 1 revealed that in the presence of an auditory sequence of tones, a frequency change of sufficient magnitude, when synchronized with the onset of the visual target, can lead to a significant improvement in visual target identification. In Experiments 2-5, we demonstrated that any abruptly onset cue that is synchronous with the presentation of the visual target also significantly improves participants' visual target identification performance. Specifically, the results of Experiment 3 showed that unimodal auditory and tactile and bimodal audiotactile cues were all equally effective in terms of improving the participants' target identification performance. On the basis of the results of Experiments 1-3, and Vroomen and de Gelder's (2000) previous research, one might have assumed that the facilitation of participants' visual target identification performance was crossmodal in nature, given that the cues used were always nonvisual. The results of Experiments 4 and 5, however, demonstrated that the presentation of a synchronous visual cue (the outline of a box surrounding the visual display) also led to a significant improvement in participants' performance on the visual target identification task.

The results of Experiment 5 revealed that both auditory and visual cues effectively facilitated the participants' visual target identification performance, although the auditory cues appeared to be more effective than the visual cues at doing so. Previous studies have also revealed the effectiveness of intramodal visual cuing on participants' visual target identification (e.g., Jonides, 1981; Klein \& Dick, 2002; Posner \& Cohen, 1984) and visual perception (Watanabe \& Shimojo, 1998). For example, Klein and Dick demonstrated that presenting a visual cue at the same time and location as the first target presented in a rapid serial visual presentation (RSVP) stream led to the more accurate identification of the visual target. It is important to note that studies such as Klein and Dick's have typically used cues that were spatially informative with regard to the likely location of the target. The results of Experiment 5, however, demonstrated that the benefits observed following the presentation of the synchronous cues need not be spatially informative nor necessarily crossmodal. Nevertheless, the reduction in RTs following the presentation of the temporally synchronous auditory cues was $752 \mathrm{msec}$, whereas the reduction following the visual cues was only $522 \mathrm{msec}$, suggesting perhaps that auditory (i.e., crossmodal) cues may still be somewhat more effective than visual (i.e., intramodal) cues in facilitating participants' visual target identification performance (see also Noesselt et al., 2008).

In their original study, Vroomen and de Gelder (2000) argued that one of the principal reasons why the LLHL sequence (as compared with the LLLL sequence) improved participants' visual target identification was because the segregation of the high tone from the low tones gave rise to visual stream segregation. Although their proposed crossmodal perceptual organization account may help explain part of the performance improvements observed when comparing LLLL with LLHL tone sequence conditions (in Experiment 1), the results of Experiments 2-5 of the present study, in which a single, abrupt, and temporally synchronous cue was synchronized with the presentation of the visual target, suggest that perhaps the two conditions (LLHL or single high tone) reflect the operation of somewhat different mechanisms. That is, it is possible, at least in theory, that the facilitation that occurs when a single tone is presented is different from the facilitation that occurs when a stream of auditory stimuli is presented.

Vroomen and de Gelder (2000) demonstrated that two key factors in determining the strength of the auditory stream segregation and, therefore, the strength of the improvement in the identification of the synchronous visual target were the abruptness and the temporal synchrony of the auditory tone with respect to the onset of the visual target display (although note that they use "abruptness" to describe the magnitude of the frequency change from the low to the high tone, rather than to describe the abrupt onset of the auditory cue). Consistent with this notion of abruptness and temporal synchrony, research by Olivers and Van der Burg (2008) has shown that the presence of a single tone whose onset was temporally synchronous with the target(s) in an RSVP stream led to a $10 \%$ improvement in participants' visual target identification performance. Importantly, Olivers and Van der Burg showed that the synchronous tone helped the second target in the RSVP stream to "escape the attentional blink" (p. 191), leading to a level of performance that was actually comparable to that for the first target in the RSVP stream.

Recently, several research groups have used the visual search paradigm in order to show that the presentation of a single auditory and/or tactile cue in synchrony with a color change in the visual target can lead to significantly shorter visual search latencies, as compared with when no cue is presented (Ngo \& Spence, 2010; Van der Burg et al., 
2008b, 2009). The results of Experiments 2 and 3 of the present study echo the findings of these aforementioned studies (e.g., Dalton \& Spence, 2007; Ngo \& Spence, 2010; Olivers \& Van der Burg, 2008; Van der Burg et al., $2008 \mathrm{~b}, 2009$ ) in showing that the presentation of abrupt, temporally synchronous nonvisual cues (be they auditory, tactile, or audiotactile) can result in faster and more accurate visual target identification performance (see also Van der Burg et al., 2010).

Analogous to the freezing effect reported by Vroomen and de Gelder (2000), the time's subjective expansion (TSE) effect put forward by Tse, Intriligator, Rivest, and Cavanagh (2004) describes the fact that low-probability, oddball stimuli (stimuli that differ from the highprobability stimuli in one or more features) tend to be perceived as having been presented for longer when presented amid a series of other high-probability stimuli. In the present study, the synchronous presentation of the cue (be it crossmodal or intramodal) with the visual target may effectively have made the target display into an oddball among the other unimodal visual distractor displays. Assuming that the perceived duration of the oddball target display was increased, this might, then, help to explain the improved identification of the visual target when it was presented at the same time as a cue, as compared with when no cue was presented. Thus, it is possible that the effect of the synchronous cue reflected some kind of enhancement of visible persistence (K.-M. Chen \& Yeh, 2009; Coltheart, 1980; Kanai, Paffen, Hogendoorn, \& Verstraten, 2006) of the target, which, in turn, may have led to an enhancement of the saliency of the visual target (Y.-C. Chen \& Yeh, 2008; Lippert, Logothetis, \& Kayser, 2007; Stein, London, Wilkinson, \& Price, 1996; Van der Burg, Olivers, Bronkhorst, \& Theeuwes, 2008a, 2008b, 2009; but see Odgaard, Arieh, \& Marks, 2003).

It is also possible that the visual or nonvisual cue merely acted as a temporal marker, signifying the exact moment in time at which the target appeared in the rapidly changing displays (Watson et al., 2003). Given that the visual target in the experiments reported here always occurred in the third position out of each four-display cycle, the synchronous cue, regardless of its sensory modality of occurrence, may have helped the participants become more attuned to the timing of the presentation of the displays. Consequently, the participants may have directed their attention to the time at which the target was expected to appear, thus leading to the speeded identification of the visual target in the presence of the synchronized cue (cf. Coull \& Nobre, 1998; Kingstone, 1992). A similar line of reasoning was used by Lippert et al. (2007). They reported that the presentation of an auditory cue improved participants' visual contrast detection performance, relative to a condition in which no cue was presented. However, this was true only when the cue provided reliable information with respect to the timing of the visual target (i.e., only if the sound always occurred at the precise moment at which the target appeared). Lippert et al. argued that the participants used their "knowledge about the timing of the auditory stimulus to focus processing on the particular instant of time highlighted by the sound" (p. 104).
We suspect that the effects observed in the presence of the temporally synchronous cues led to an enhanced representation (in terms of either visible persistence or saliency) of the visual target (K.-M. Chen \& Yeh, 2009; Y.-C. Chen \& Yeh, 2008; Lippert et al., 2007; Noesselt et al., 2008; Pascucci, Megna, Panichi, \& Baldassi, 2009; Prinzmetal, McCool, \& Park, 2005; Stein et al., 1996; Van der Burg et al., 2008a, 2008b, 2009). Vroomen and de Gelder (2000) stated that their participants reported the visual target to freeze for a short period of time in the presence of the segregated high tone. Van der Burg et al. $(2008 b, 2009)$, on the other hand, reported that their participants described the visual target as popping out from among the distractors. At present, it is impossible to say whether any such enhancement of the target representation primarily affected the perceived duration and/or saliency of the target (note that this distinction might map on to the subjectively reported distinction between the freezing phenomenon and the visual target's popping out). The oddball account may explain why there was a reported perceptual freezing effect, or TSE, in which the participants perceived the duration of the target display to be longer than the distractor displays. The temporal marker account may offer a plausible explanation for the reported pop-out of the target among the distractors, which, in turn, may have manifested in the observed improvements in both the speed and accuracy of participants' visual target identification performance.

Although the descriptions of the visual target as freezing or popping-out seem to be phenomenally different, with the former reflecting an enhancement in the duration and the latter reflecting an enhancement in the saliency of the visual target, it is also possible that the effect that a temporally synchronous nonvisual cue has on a visual target in each case may be the same phenomenally but that the use of either description (e.g., by participants and/or experimenters) may simply depend on the task, stimuli, and experimental paradigm used. For example, in Vroomen and de Gelder's (2000) study, just as in the present study, the visual target and distractors were rapidly presented and masked. The very brief presentation of the visual target is what, presumably, made the participants' task so difficult. It would, therefore, probably have benefited participants most if the synchronous tone were somehow able to freeze the frame of the target, allowing them to continue to access the information contained within the target frame for a short but extended period of time. In Van der Burg et al.'s (2008b, 2009) studies, on the other hand, participants searched for the visual target among a dynamic and cluttered field of distractors. In this case, the participants would have benefited the most from having a synchronous cue that could make the visual target more salient than the distractors (i.e., making the visual target pop out from among the surrounding distractor stimuli). In this case, it is possible that the synchronous cue somehow results in the enhancement of the saliency of the visual target (Y.-C. Chen \& Yeh, 2008; Lippert et al., 2007; Stein et al., 1996; Van der Burg et al., 2008a, 2008b, 2009; although see Odgaard et al., 2003). Rather than reflecting the operation of different mechanisms, however, it may very well be the 
case that whether participants report the visual target as freezing or popping out when presented in synchrony with an abrupt cue depends on the descriptors that are most appropriate or beneficial for the task and stimuli at hand.

Whatever the correct interpretation of the effects reported here, our findings nevertheless help shed light on the mechanisms underlying the facilitation of participants' visual target identification performance when a synchronous cue is presented with the target in a rapidly masked stream of visual stimuli. Taken together, the results of the present study both replicate (Experiment 1) and extend (Experiments 2-5) Vroomen and de Gelder's (2000) findings by demonstrating that a single, abrupt, and temporally synchronous cue (auditory, tactile, audiotactile, or visual) can give rise to significant improvements in participants' visual target identification performance, and they also reveal that the observed benefits are not limited to crossmodal cues.

\section{AUTHOR NOTE}

M.K.N. was supported by a Clarendon Fund Scholarship from the University of Oxford, Oxford, U.K. Correspondence concerning this article should be addressed to M. K. Ngo, Crossmodal Research Laboratory, Department of Experimental Psychology, University of Oxford, South Parks Road, OX1 3UD Oxford, England (e-mail: thuan.ngo@) psy.ox.ac.uk).

\section{REFERENCES}

Anderson, T. S., \& Mamassian, P. (2008). Audiovisual integration of stimulus transients. Vision Research, 48, 2537-2544.

Bregman, A. S. (1990). Auditory scene analysis. Cambridge, MA: MIT Press.

Chen, K.-M., \& YeH, S.-L. (2009). Asymmetric cross-modal effects in time perception. Acta Psychologica, 130, 225-234.

Chen, Y.-C., \& Yeh, S.-L. (2008). Visual events modulated by sound in repetition blindness. Psychonomic Bulletin \& Review, 15, 404-408.

Coltheart, M. (1980). Iconic memory and visible persistence. Perception \& Psychophysics, 27, 183-228.

Coull, J. T., \& Nobre, A. C. (1998). Where and when to pay attention: The neural systems for directing attention to spatial locations and to time intervals as revealed by both PET and fMRI. Journal of Neuroscience, 18, 7426-7435.

Dalton, P., \& Spence, C. (2007). Attentional capture in serial audiovisual search tasks. Perception \& Psychophysics, 69, 422-438.

JoNIDES, J. (1981). Voluntary versus automatic control over the mind's eye's movement. In J. Long \& A. Baddeley (Eds.), Attention and performance $I X$ (pp. 187-203). Hillsdale, NJ: Erlbaum.

Kanai, R., Paffen, C. L. E., Hogendoorn, H., \& Verstraten, F. A. J. (2006). Time dilation in dynamic visual display. Journal of Vision, $\mathbf{6}$, 1421-1430.

Kingstone, A. (1992). Combining expectancies. Quarterly Journal of Experimental Psychology, 44A, 69-104.

Klein, R. M., \& Dick, B. (2002). Temporal dynamics of reflexive attention shifts: A dual-stream rapid serial visual presentation exploration. Psychological Science, 13, 176-179.

Leber, A. B., KaWahara, J.-I., \& Gabari, Y. (2009). Long-term abstract learning of attentional set. Journal of Experimental Psychology: Human Perception \& Performance, 35, 1385-1397.

Lippert, M., Logothetis, N. K., \& Kayser, C. (2007). Improvement of visual contrast detection by a simultaneous sound. Brain Research, 1173, 102-109.

NGo, M. K., \& SPENCE, C. (2010). Auditory, tactile, and multisensory cues can facilitate search for dynamic visual stimuli. Attention, Perception, \& Psychophysics, 72, 1654-1665.

Noesselt, T., Bergmann, D., Hake, M., Heinze, H.-J., \& FenDRICH, R. (2008). Sound increases the saliency of visual events. Brain Research, 1220, 157-163.
Odgaard, E. C., Arieh, Y., \& Marks, L. E. (2003). Cross-modal enhancement of perceived brightness: Sensory interaction versus response bias. Perception \& Psychophysics, 65, 123-132.

O'Leary, A., \& Rhodes, G. (1984). Cross-modal effects on visual and auditory object perception. Perception \& Psychophysics, 35, 565-569.

Olivers, C. N. L., \& VAN DER BURG, E. (2008). Bleeping you out of the blink: Sound saves vision from oblivion. Brain Research, 1242, 191-199.

Pascucci, D., Megna, N., Panichi, M., \& Baldassi, S. (2009). How does sound improve vision? A classification image study. Perception, 38, 18.

Posner, M. I., \& Cohen, Y. (1984). Components of performance. In H. Bouma \& D. G. Bouwhuis (Eds.), Attention and performance X: Control of language processes (pp. 531-556). Hillsdale, NJ: Erlbaum.

Prinzmetal, W., McCool, C., \& Park, S. (2005). Attention: Reaction time and accuracy reveal different mechanisms. Journal of Experimental Psychology: General, 134, 73-92.

Santangelo, V., Ho, C., \& Spence, C. (2008). Capturing spatial attention with multisensory cues. Psychonomic Bulletin \& Review, 15, 398-403.

SPence, C., \& Chen, Y.-C. (in press). Intramodal and crossmodal perceptual grouping. In B. E. Stein et al. (Eds.), The new handbook of multisensory processing. Cambridge, MA: MIT Press.

Spence, C., Sanabria, D., \& Soto-Faraco, S. (2007). Intersensory Gestalten and crossmodal scene perception. In K. Noguchi (Ed.), Psychology of beauty and Kansei: New horizons of Gestalt perception (pp. 519-579). Tokyo: Fuzanbo International.

Spence, C., \& Santangelo, V. (2009). Capturing spatial attention with multisensory cues: A review. Hearing Research, 258, 134-142.

Stein, B. E., London, N., Wilkinson, L. K., \& Price, D. P. (1996). Enhancement of perceived visual intensity by auditory stimuli: A psychophysical analysis. Journal of Cognitive Neuroscience, 8, 497-506.

Tse, P. U., Intriligator, J., Rivest, J., \& Cavanagh, P. (2004). Attention and the subjective expansion of time. Perception \& Psychophysics, 66, 1171-1189.

Van der Burg, E., Cass, J., Olivers, C. N. L., Theeuwes, J., \& ALAIS, D. (2010). Efficient visual search from synchronized auditory signals requires transient audiovisual events. PLOS ONE, 5, e10664. doi:10.1371/journal.pone. 001066

Van der Burg, E., Olivers, C. N. L., Bronkhorst, A. W., \& TheeuWES, J. (2008a). Audiovisual events capture attention: Evidence from temporal order judgments. Journal of Vision, 8(5, Art. 2), 1-10.

VAn der Burg, E., Olivers, C. N. L., Bronkhorst, A. W., \& TheeuWES, J. (2008b). Pip and pop: Nonspatial auditory signals improve spatial visual search. Journal of Experimental Psychology: Human Perception \& Performance, 34, 1053-1065.

Van der Burg, E., Olivers, C. N. L., Bronkhorst, A. W., \& TheeuWES, J. (2009). Poke and pop: Tactile-visual synchrony increases visual saliency. Neuroscience Letters, 450, 60-64.

VRoomen, J., \& de Gelder, B. (2000). Sound enhances visual perception: Cross-modal effects of auditory organization on vision. Journal of Experimental Psychology: Human Perception \& Performance, 26, $1583-1590$.

Watanabe, K., \& Shimojo, S. (1998). Attentional modulation in perception of visual motion events. Perception, 27, 1041-1054.

Watanabe, K., \& Shimojo, S. (2001). When sound affects vision: Effects of auditory grouping on visual motion perception. Psychological Science, 12, 109-116.

Watson, D. G., Humphreys, G. W., \& Olivers, C. N. L. (2003). Visual marking: Using time in visual selection. Trends in Cognitive Sciences, 7, 180-186.

\section{NOTE}

1. Vroomen and de Gelder (2000) used the number of targets shown (NTS) as their measure of the speed of participants' target identification responses. For comparison, in the present study, the averages for NTS were 3.3 and 2.5 for the tone-absent and tone-present conditions, respectively. This compares with 3.3 for the LLLL and 2.9 for the LLHL conditions in Experiment 1 of Vroomen and de Gelder's study. 